\title{
Application of Residual Power Series Method to Fractional Coupled Physical Equations Arising in Fluids Flow
}

\author{
Anas Arafa ${ }^{1}$ and Ghada Elmahdy $\mathbb{D}^{2}$ \\ ${ }^{1}$ Department of Mathematics and Computer Science, Faculty of Science, Port Said University, Port Said, Egypt \\ ${ }^{2}$ Department of Basic science, Canal High Institute of Engineering and Technology, Suez, Egypt \\ Correspondence should be addressed to Ghada Elmahdy; ghada.elmahdy91@gmail.com
}

Received 28 March 2018; Accepted 3 June 2018; Published 4 July 2018

Academic Editor: Julio D. Rossi

Copyright (c) 2018 Anas Arafa and Ghada Elmahdy. This is an open access article distributed under the Creative Commons Attribution License, which permits unrestricted use, distribution, and reproduction in any medium, provided the original work is properly cited.

\begin{abstract}
The approximate analytical solution of the fractional Cahn-Hilliard and Gardner equations has been acquired successfully via residual power series method (RPSM). The approximate solutions obtained by RPSM are compared with the exact solutions as well as the solutions obtained by homotopy perturbation method (HPM) and q-homotopy analysis method (q-HAM). Numerical results are known through different graphs and tables. The fractional derivatives are described in the Caputo sense. The results light the power, efficiency, simplicity, and reliability of the proposed method.
\end{abstract}

\section{Introduction}

Fractional differential equations (FDEs) have found applications in many problems in physics and engineering [1, 2]. Since most of the nonlinear FDEs cannot be solved exactly, approximate and numerical methods must be used. Some of the recent analytical methods for solving nonlinear problems include the Adomian decomposition method [3, 4], variational iteration method [5], homotopy perturbation method [6, 7], homotopy analysis method [8,9], spectral collocation method [10], the tanh-coth method [11], expfunction method [12], Mittag-Leffler function method [13], differential quadrature method [14], and reproducing kernel Hilbert space method $[15,16]$.

The Gardner equation [17] (combined KdV-mKdV equation) is a useful model for the description of internal solitary waves in shallow water,

$$
u_{t}+6 u u_{x} \pm 6 u^{2} u_{x}+u_{x x x}=0 .
$$

Those two models will be classified as positive Gardner equation and negative Gardner equation depending on the sign of the cubic nonlinear term [18, 19]. Gardner equation is widely used in various branches of physics, such as plasma physics, fluid physics, and quantum field theory [20, 21]. It also describes a variety of wave phenomena in plasma and solid state $[22,23]$.

The Cahn-Hilliard equation [24] is one type of partial differential equations (PDEs) and was first introduced in 1958 as a model for process of phase separation of a binary alloy under the critical temperature [25],

$$
u_{t}=\gamma u_{x}+6 u u_{x}^{2}+\left(3 u^{2}-1\right) u_{x x}-u_{x x x x}, \quad \gamma \geq 0 .
$$

This equation is related to a number of interesting physical phenomena like the spinodal decomposition, phase separation, and phase ordering dynamics. On the other hand it becomes important in material sciences $[26,27]$.

The aim of this paper is to study the time-fractional Gardner equation [28-30] and time-fractional Cahn-Hilliard equation [31-37] of this form,

$$
\begin{array}{r}
D_{t}^{\alpha} u(x, t)+6\left(u-\varepsilon^{2} u^{2}\right) u_{x}+u_{x x x}=0, \\
D_{t}^{\alpha} u(x, t)-u_{x}-6 u u_{x}^{2}-\left(3 u^{2}-1\right) u_{x x}+u_{x x x x}=0,
\end{array}
$$

where $0<\alpha \leq 1,-\infty<x<\infty$, and $0 \leq t<R$. Numerous methods have been used to solve this equations, for example, q-Homotopy analysis method [28], the new version of F-expansion method [29], reduced differential transform 
method [30], the generalized tanh-coth method [38], the generalized Kudryashov method [39], extended fractional Riccati expansion method [31], subequation method [32], homotopy analysis method [33], the Adomian decomposition method [34], improved $(\grave{G} / G)$-expansion method [35], homotopy perturbation method [36], and variational iteration method [37]. We solve Cahn-Hilliard equation and Gardner equation by RPSM.

The RPSM was first devised in 2013 by the Jordanian mathematician Omar Abu Arqub as an efficient method for determining values of coefficients of the power series solution for first and the second-order fuzzy differential equations [40]. The RPSM is an effective and easy to construct power series solution for strongly linear and nonlinear equations without linearization, perturbation, or discretization. In the last few years, the RPSM has been applied to solve a growing number of nonlinear ordinary and PDEs of different types, classifications, and orders. It has been successfully applied in the numerical solution of the generalized Lane-Emden equation [41], which is a highly nonlinear singular differential equation, in the numerical solution of higher-order regular differential equations [42], in approximate solution of the nonlinear fractional KdV-Burgers equation [43], in construct and predict the solitary pattern solutions for nonlinear time-fractional dispersive PDEs [44], and in predicting and representing the multiplicity of solutions to boundary value problems of fractional order [45]. The RPSM distinguishes itself from various other analytical and numerical methods in several important aspects [46]. Firstly, the RPSM does not need to compare the coefficients of the corresponding terms and a recursion relation is not required. Secondly, the RPSM provides a simple way to ensure the convergence of the series solution by minimizing the related residual error. Thirdly, the RPSM is not affected by computational rounding errors and does not require large computer memory and time. Fourthly, the RPSM does not require any converting while switching from the low-order to the higher-order and from simple linearity to complex nonlinearity; as a result, the method can be applied directly to the given problem by choosing an appropriate initial guess approximation.

\section{Fundamental Concepts}

Definition 1 (see [43]). The Caputo time-fractional derivatives of order $\alpha>0$ of $u(x, t)$ is defined as

$$
\begin{aligned}
& D_{t}^{\alpha} u(x, t) \\
& = \begin{cases}\frac{1}{\Gamma_{(n-\alpha)}} \int_{0}^{t}(t-\tau)^{n-\alpha-1} \frac{\partial^{n} u(x, \tau)}{\partial \tau^{n}} d \tau, & n-1<\alpha<n, \\
\frac{\partial^{n} u(x, t)}{\partial t^{n}}, & \alpha=n \in N .\end{cases}
\end{aligned}
$$

Definition 2 (see $[47,48])$. A power series representation of the form

$$
\begin{aligned}
\sum_{n=0}^{\infty} C_{n}\left(t-t_{0}\right)^{n \alpha}= & C_{0}+C_{1}\left(t-t_{0}\right)^{\alpha}+C_{2}\left(t-t_{0}\right)^{2 \alpha} \\
& +\ldots
\end{aligned}
$$

where $0 \leq n-1<\alpha \leq n, n \in N$ and $t \geq t_{0}$ is called fractional power series about $t_{0}$.

Theorem 3 (see [47, 48]). Suppose that $f$ has a fractional power series representation at $t_{0}$ of the form

$$
\begin{aligned}
f(t)= & \sum_{n=0}^{\infty} C_{n}\left(t-t_{0}\right)^{n \alpha}, \\
& \text { where } 0 \leq n-1<\alpha \leq n \text { and } t_{0} \leq t<t_{0}+R .
\end{aligned}
$$

If $D^{n \alpha} f(t)$ are continuous on $\left(t_{0}, t_{0}+R\right), n=0,1,2,3, \ldots$, then coefficients $C_{n}$ will take the form

$$
C_{n}=\frac{D^{n \alpha} f\left(t_{0}\right)}{\Gamma(n \alpha+1)} .
$$

Definition 4 (see [43]). A power series representation of the form

$$
\begin{aligned}
\sum_{n=0}^{\infty} f_{n}(x)\left(t-t_{0}\right)^{n \alpha}= & f_{0}(x)+f_{1}(x)\left(t-t_{0}\right)^{\alpha} \\
& +f_{2}(x)\left(t-t_{0}\right)^{2 \alpha}+\ldots
\end{aligned}
$$

is called a multiple fractional power series about $t=t_{0}$.

Theorem 5 (see $[43,44]$ ). Suppose that $u(x, t)$ has a multiple fractional Power series representation at $t_{0}$ of the form

$$
u(x, t)=\sum_{n=0}^{\infty} f_{n}(x)\left(t-t_{0}\right)^{n \alpha}
$$

where $x \in I, 0 \leq n-1<\alpha \leq n$ and $t_{0} \leq t<t_{0}+R$.

If $D_{t}^{n \alpha} u(x, t)$ are continuous on $I \times\left(t_{0}, t_{0}+R\right), n=0,1,2,3, \ldots$, then coefficients $f_{n}(x)$ will take the form

$$
f_{n}(x)=\frac{D_{t}^{n \alpha} u\left(x, t_{0}\right)}{\Gamma(n \alpha+1)} .
$$

Corollary 6 (see [44]). Suppose that $u(x, y, t)$ has a multiple fractional Power series representation at $t_{0}$ of the form

$$
\begin{aligned}
& u(x, y, t)=\sum_{n=0}^{\infty} f_{n}(x, y)\left(t-t_{0}\right)^{n \alpha}, \\
& \quad(x, y) \in I_{1} \times I_{2}, t_{0} \leq t<t_{0}+R .
\end{aligned}
$$

If $D_{t}^{n \alpha} u(x, y, t)$ are continuous on $I_{1} \times I_{2} \times\left(t_{0}, t_{0}+R\right), n=$ $0,1,2,3, \ldots$, then $f_{n}(x, y)$ will take the form

$$
f_{n}(x, y)=\frac{D_{t}^{n \alpha} u\left(x, y, t_{0}\right)}{\Gamma(n \alpha+1)} .
$$

\section{Basic Idea of RPSM}

To give the approximate solution of nonlinear fractional order differential equations by means of the RPSM, we consider a general nonlinear fractional differential equation:

$$
D^{\alpha} u(x, t)=N(u)+R(u)
$$


where $N(u)$ is nonlinear term and $R(u)$ is a linear term. Subject to the initial condition

$$
u(x, 0)=f(x) .
$$

The RPSM proposes the solution for (14) as a fractional power series about the initial point $t=0$,

$$
\begin{aligned}
u(x, t)=\sum_{n=0}^{\infty} f_{n}(x) & \frac{t^{n \alpha}}{\Gamma(1+n \alpha)}, \\
0 & <\alpha \leq 1,-\infty<x<\infty, 0 \leq t<R .
\end{aligned}
$$

Next we let $u_{k}(x, t)$ denote the kth truncated series of $u(x, t)$,

$$
u_{k}(x, t)=\sum_{n=0}^{k} f_{n}(x) \frac{t^{n \alpha}}{\Gamma(1+n \alpha)} .
$$

The 0th RPS approximate solution of $u(x, t)$ is

$$
u_{0}(x, t)=u(x, 0)=f(x) .
$$

Equation (17) can be written as

$$
\begin{aligned}
u_{k}(x, t)=f(x)+\sum_{n=1}^{k} f_{n}(x) \frac{t^{n \alpha}}{\Gamma(1+n \alpha)}, & \\
& k=1,2,3, \ldots .
\end{aligned}
$$

We define the residual function for (14)

$$
\operatorname{Res}_{u}(x, t)=D_{t}^{\alpha} u(x, t)-N(u)-R(u) .
$$

Therefore, the kth residual function $\operatorname{Res}_{u, k}$ is

$$
\operatorname{Res}_{u, k}(x, t)=D_{t}^{\alpha} u_{k}(x, t)-N\left(u_{k}\right)-R\left(u_{k}\right) .
$$

As in [40, 41], $\operatorname{Resu}(x, t)=0$ and $\lim _{k \rightarrow \infty} \operatorname{Res}_{k}(x, t)=$ $\operatorname{Res}(x, t)$.Therefore, $D_{t}^{n \alpha} \operatorname{Res}(x, t)=0$ since the fractional derivative of a constant in the Caputo sense is zero and the fractional derivatives $D_{t}^{n \alpha}$ of $\operatorname{Res}(x, t)$ and $\operatorname{Res}_{k}(x, t)$ are matching at $t=0$ for each $n=0,1,2, \ldots, k$; that is, $D_{t}^{n \alpha} \operatorname{Res}(x, 0)=D_{t}^{n \alpha} \operatorname{Res}_{k}(x, 0)=0, n=0,1,2, \ldots, k$.

To determine $f_{1}(x), f_{2}(x), f_{3}(x), \ldots$ we consider $k=$ $1,2,3, .$. in (19) and substitute it into (21), applying the fractional derivative $D_{t}^{(k-1) \alpha}$ in both sides, $k=1,2,3, \ldots$, and finally we solve

$$
D_{t}^{(k-1) \alpha} \operatorname{Res}_{u, k}(x, 0)=0, \quad k=1,2,3, \ldots
$$

\section{Applications}

To illustrate the basic idea of RPSM, we consider the following two time-fractional Gardner and Cahn-Hilliard equations.

4.1. Time-Fractional Gardner Equation. Consider the timefractional homogeneous Gardner equation

$$
D_{t}^{\alpha} u(x, t)+6\left(u-\varepsilon^{2} u^{2}\right) u_{x}+u_{x x x}=0 .
$$

Subject to the initial Condition

$$
u(x, 0)=\frac{1}{2}+\frac{1}{2} \tanh \left[\frac{x}{2}\right] .
$$

The exact solution when $\varepsilon=1, \alpha=1$ is

$$
u(x, t)=\frac{1}{2}+\frac{1}{2} \tanh \left[\frac{x-t}{2}\right] .
$$

We define the residual function for (23) as

$$
\operatorname{Res}_{u}(x, t)=D_{t}^{\alpha} u(x, t)+6\left(u-\varepsilon^{2} u^{2}\right) u_{x}+u_{x x x},
$$

therefore, for the kth residual function $\operatorname{Res}_{u, k}(x, t)$,

$$
\operatorname{Res}_{u, k}(x, t)=D_{t}^{\alpha} u_{k}+6\left(u_{k}-\varepsilon^{2} u_{k}^{2}\right) u_{k x}+u_{k x x x} .
$$

To determine $f_{1}(x)$, we consider $(k=1)$ in (27)

$$
\operatorname{Res}_{u, 1}(x, t)=D_{t}^{\alpha} u_{1}+6 u_{1} u_{1 x}-6 \varepsilon^{2} u_{1}^{2} u_{1 x}+u_{1 x x x} .
$$

But from (19) at $k=1$,

$$
\begin{aligned}
& u_{1}(x, t)=f(x)+f_{1}(x) \frac{t^{\alpha}}{\Gamma(1+\alpha)}, \\
& \operatorname{Res}_{u, 1}(x, t)=f_{1}+6 f f_{x}-6 \varepsilon^{2} f_{x} f^{2}+f_{x x x}+\left[6 f f_{1 x}\right. \\
& \left.\quad+6 f_{1} f_{x}-12 \varepsilon^{2} f_{x} f f_{1}-6 \varepsilon^{2} f_{1 x} f^{2}+f_{1 x x x}\right] \\
& \cdot \frac{t^{\alpha}}{\Gamma(1+\alpha)}+\left[6 f_{1} f_{1 x}-6 \varepsilon^{2} f_{x} f_{1}^{2}-12 \varepsilon^{2} f_{1 x} f f_{1}\right] \\
& \cdot \frac{t^{2 \alpha}}{\Gamma(1+\alpha)^{2}}-6 \varepsilon^{2} f_{1 x} f_{1}^{2} \frac{t^{3 \alpha}}{\Gamma(1+\alpha)^{3}} .
\end{aligned}
$$

Now depending on the result of (22) In the case of $k=1$, we have $\operatorname{Res}_{u_{1}}(x, 0)=0$,

$$
\begin{aligned}
& f_{1}=-6 f f_{x}+6 \varepsilon^{2} f_{x} f^{2}-f_{x x x}, \\
& f_{1}(x)=\frac{1}{8} \operatorname{sech}\left[\frac{x}{2}\right]^{4}\left(-1+\left(-4+3 \varepsilon^{2}\right) \cosh [x]\right. \\
& \left.\quad+3\left(-1+\varepsilon^{2}\right) \sinh [x]\right) .
\end{aligned}
$$

To determine $f_{2}(x)$, we consider $(k=2)$ in (27)

$$
\operatorname{Res}_{u, 2}(x, t)=D_{t}^{\alpha} u_{2}+6 u_{2} u_{2 x}-6 \varepsilon^{2} u_{2}^{2} u_{2 x}+u_{2 x x x} .
$$


But from (19) at $k=2$,

$$
\begin{aligned}
& u_{2}(x, t)=f(x)+f_{1}(x) \frac{t^{\alpha}}{\Gamma(1+\alpha)}+f_{2}(x) \\
& \frac{t^{2 \alpha}}{\Gamma(1+2 \alpha)} \\
& \operatorname{Res}_{u, 2}(x, t)=f_{1}+6 f f_{x}-6 \varepsilon^{2} f_{x} f^{2}+f_{x x x}+\left[f_{2}\right. \\
& \left.+6 f f_{1 x}+6 f_{1} f_{x}-12 \varepsilon^{2} f_{x} f f_{1}-6 \varepsilon^{2} f_{1 x} f^{2}+f_{1 x x x}\right] \\
& \cdot \frac{t^{\alpha}}{\Gamma(1+\alpha)}+\left[6 f f_{2 x}+6 f_{2} f_{x}-12 \varepsilon^{2} f_{x} f f_{2}\right. \\
& \left.-6 \varepsilon^{2} f_{2 x} f^{2}+f_{2 x x x}\right] \frac{t^{2 \alpha}}{\Gamma(1+2 \alpha)}+\left[6 f_{1} f_{1 x}\right. \\
& \left.-6 \varepsilon^{2} f_{x} f_{1}^{2}-12 \varepsilon^{2} f_{1 x} f f_{1}\right] \frac{t^{2 \alpha}}{\Gamma(1+\alpha)^{2}}+\left[6 f_{1} f_{2 x}\right. \\
& +6 f_{2} f_{1 x}-12 \varepsilon^{2} f_{x} f_{1} f_{2}-12 \varepsilon^{2} f_{1 x} f f_{2} \\
& \left.-12 \varepsilon^{2} f_{2 x} f f_{1}\right] \frac{t^{3 \alpha}}{\Gamma(1+\alpha) \Gamma(1+2 \alpha)}-6 \varepsilon^{2} f_{1 x} f_{1}^{2} \\
& \cdot \frac{t^{3 \alpha}}{\Gamma(1+\alpha)^{3}}+\left[6 f_{2} f_{2 x}-6 \varepsilon^{2} f_{x} f_{2}^{2}-12 \varepsilon^{2} f_{2 x} f f_{2}\right] \\
& \frac{t^{4 \alpha}}{\Gamma(1+2 \alpha)^{2}}+\left[-12 \varepsilon^{2} f_{1 x} f_{1} f_{2}-6 \varepsilon^{2} f_{2 x} f_{1}^{2}\right] \\
& \frac{t^{4 \alpha}}{\Gamma(1+\alpha)^{2} \Gamma(1+2 \alpha)}+\left[-6 \varepsilon^{2} f_{1 x} f_{2}^{2}\right. \\
& \left.-12 \varepsilon^{2} f_{2 x} f_{1} f_{2}\right] \frac{t^{5 \alpha}}{\Gamma(1+\alpha) \Gamma(1+2 \alpha)^{2}}-6 \varepsilon^{2} f_{2 x} f_{2}^{2} \\
& \frac{t^{6 \alpha}}{\Gamma(1+2 \alpha)^{3}}
\end{aligned}
$$

Applying $D_{t}^{\alpha}$ on both sides and solving the equation $D_{t}^{\alpha} \operatorname{Res}_{u, 2}(x, 0)=0$, then we get

$$
\begin{aligned}
f_{2} & =-6 f f_{1 x}-6 f_{1} f_{x}+12 \varepsilon^{2} f_{x} f f_{1}+6 \varepsilon^{2} f_{1 x} f^{2} \\
& -f_{1 x x x}, \\
f_{2} & (x)=\frac{-1}{64} \operatorname{sech}\left[\frac{x}{2}\right]^{7}\left(-24\left(-1+\varepsilon^{2}\right) \cosh \left[\frac{x}{2}\right]\right. \\
& -6\left(22-37 \varepsilon^{2}+15 \varepsilon^{4}\right) \cosh \left[\frac{3 x}{2}\right]+24 \cosh \left[\frac{5 x}{2}\right] \\
& -42 \varepsilon^{2} \cosh \left[\frac{5 x}{2}\right]+18 \varepsilon^{4} \cosh \left[\frac{5 x}{2}\right] \\
& +206 \sinh \left[\frac{x}{2}\right]-204 \varepsilon^{2} \sinh \left[\frac{x}{2}\right] \\
& -129 \sinh \left[\frac{3 x}{2}\right]+222 \varepsilon^{2} \sinh \left[\frac{3 x}{2}\right]
\end{aligned}
$$

$$
\begin{aligned}
& -90 \varepsilon^{4} \sinh \left[\frac{3 x}{2}\right]+25 \sinh \left[\frac{5 x}{2}\right] \\
& \left.-42 \varepsilon^{2} \sinh \left[\frac{5 x}{2}\right]+18 \varepsilon^{4} \sinh \left[\frac{5 x}{2}\right]\right) .
\end{aligned}
$$

The solution in series form is given by

$$
\begin{aligned}
u(x, t)= & f(x)+f_{1}(x) \frac{t^{\alpha}}{\Gamma(1+\alpha)}+f_{2}(x) \frac{t^{2 \alpha}}{\Gamma(1+2 \alpha)} \\
& +\ldots
\end{aligned}
$$

4.2. Time-Fractional Cahn-Hilliard Equation. Consider the time-fractional Cahn-Hilliard equation

$$
D_{t}^{\alpha} u(x, t)-u_{x}-6 u u_{x}^{2}-\left(3 u^{2}-1\right) u_{x x}+u_{x x x x}=0 .
$$

Subject to the initial condition

$$
u(x, 0)=\tanh \left[\frac{\sqrt{2}}{2} x\right] .
$$

The exact solution when $\alpha=1$ is

$$
u(x, t)=\tanh \left[\frac{\sqrt{2}}{2}(x+t)\right] .
$$

We define the residual function for (39) as

$$
\begin{aligned}
\operatorname{Res}_{u}(x, t)= & D_{t}^{\alpha} u(x, t)-u_{x}-6 u u_{x}^{2}-\left(3 u^{2}-1\right) u_{x x} \\
& +u_{x x x x},
\end{aligned}
$$

therefore, for the kth residual function $\operatorname{Res}_{u, k}(x, t)$,

$$
\begin{aligned}
\operatorname{Res}_{u, k}(x, t)= & D_{t}^{\alpha} u_{k}-u_{k x}-6 u_{k} u_{k x}^{2}-\left(3 u_{k}^{2}-1\right) u_{k x x} \\
& +u_{k x x x x} .
\end{aligned}
$$

To determine $f_{1}(x)$, we consider $(k=1)$ in (43)

$$
\begin{aligned}
\operatorname{Res}_{u, 1}(x, t)= & D_{t}^{\alpha} u_{1}-u_{1 x}-6 u_{1} u_{1 x}^{2}-\left(3 u_{1}^{2}-1\right) u_{1 x x} \\
& +u_{1 x x x x} .
\end{aligned}
$$

From (19) at $k=1$,

$$
\begin{aligned}
& u_{1}(x, t)=f(x)+f_{1}(x) \frac{t^{\alpha}}{\Gamma(1+\alpha)}, \\
& \operatorname{Res}_{u, 1}(x, t)=f_{1}-f_{x}-6 f f_{x}^{2}-3 f^{2} f_{x x}+f_{x x} \\
& \quad+f_{x x x x}+\left[-f_{1 x}-6 f_{1} f_{x}^{2}-12 f f_{x} f_{1 x}-6 f f_{1} f_{x x}\right. \\
& \left.\quad-3 f^{2} f_{1 x x}+f_{1 x x}+f_{1 x x x x}\right] \frac{t^{\alpha}}{\Gamma(1+\alpha)}+\left[-6 f f_{1 x}^{2}\right. \\
& \left.\quad-12 f_{1} f_{x} f_{1 x}-3 f_{1}^{2} f_{x x}-6 f f_{1} f_{1 x x}\right] \frac{t^{2 \alpha}}{\Gamma(1+\alpha)^{2}} \\
& \quad+\left[-6 f_{1} f_{1 x}^{2}-3 f_{1}^{2} f_{1 x x}\right] \frac{t^{3 \alpha}}{\Gamma(1+\alpha)^{3}} .
\end{aligned}
$$


If we put $\operatorname{Res}_{u, 1}(x, 0)=0$, then

$$
\begin{aligned}
& f_{1}(x)=f_{x}+6 f(x) f_{x}^{2}+3 f^{2} f_{x x}-f_{x x}-f_{x x x x}, \\
& f_{1}(x)=\frac{\operatorname{sech}[x / \sqrt{2}]^{2}}{\sqrt{2}} .
\end{aligned}
$$

Similarity, to determine $f_{2}(x)$, we substitute

$$
\begin{aligned}
u_{2}(x, t)= & f(x)+f_{1}(x) \frac{t^{\alpha}}{\Gamma(1+\alpha)} \\
& +f_{2}(x) \frac{t^{2 \alpha}}{\Gamma(1+2 \alpha)},
\end{aligned}
$$

into (43) where $k=2$,

$$
\begin{aligned}
& \operatorname{Res}_{u, 2}(x, t)=\left[f_{1}-f_{x}-6 f f_{x}^{2}-3 f^{2} f_{x x}+f_{x x}\right. \\
& \left.+f_{x x x x}\right]+\left[f_{2}-f_{1 x}-6 f_{1} f_{x}^{2}-12 f f_{x} f_{1 x}\right. \\
& \left.-6 f f_{1} f_{x x}-3 f^{2} f_{1 x x}+f_{1 x x}+f_{1 x x x x}\right] \frac{t^{\alpha}}{\Gamma(1+\alpha)} \\
& +\left[-f_{2 x}-12 f f_{x} f_{2 x}-6 f_{2} f_{x}^{2}-6 f f_{2} f_{x x}\right. \\
& \left.-3 f_{2 x x} f^{2}+f_{2 x x}+f_{2 x x x x}\right] \frac{t^{2 \alpha}}{\Gamma(1+2 \alpha)}+\left[-6 f f_{1 x}^{2}\right. \\
& \left.-12 f_{1} f_{x} f_{1 x}-3 f_{1}^{2} f_{x x}-6 f f_{1} f_{1 x x}\right] \frac{t^{2 \alpha}}{\Gamma(1+\alpha)^{2}} \\
& +\left[-12 f f_{1 x} f_{2 x}-12 f_{2} f_{x} f_{1 x}-6 f_{1} f_{2} f_{x x}\right. \\
& \left.-6 f f_{2} f_{1 x x}-12 f_{1} f_{x} f_{2 x}-6 f_{2 x x} f f_{1}\right] \\
& \cdot \frac{t^{3 \alpha}}{\Gamma(1+\alpha) \Gamma(1+2 \alpha)}+\left[-6 f_{1} f_{1 x}^{2}-3 f_{1}^{2} f_{1 x x}\right] \\
& \frac{t^{3 \alpha}}{\Gamma(1+\alpha)^{3}}+\left[-6 f f_{2 x}^{2}-12 f_{2} f_{x} f_{2 x}-3 f_{2}^{2} f_{x x}\right. \\
& \left.-6 f_{2 x x} f f_{2}\right] \frac{t^{4 \alpha}}{\Gamma(1+2 \alpha)^{2}}+\left[-12 f_{1} f_{1 x} f_{2 x}-6 f_{2} f_{1 x}^{2}\right. \\
& \left.-6 f_{1} f_{2} f_{1 x x}-3 f_{2 x x} f_{1}^{2}\right] \frac{t^{4 \alpha}}{\Gamma(1+\alpha)^{2} \Gamma(1+2 \alpha)} \\
& +\left[-6 f_{1} f_{2 x}^{2}-12 f_{2} f_{1 x} f_{2 x}-3 f_{2}^{2} f_{1 x x}-6 f_{2 x x} f_{1} f_{2}\right] \\
& \cdot \frac{t^{5 \alpha}}{\Gamma(1+\alpha) \Gamma(1+2 \alpha)^{2}}+\left[-6 f_{2} f_{2 x}^{2}-3 f_{2 x x} f_{2}^{2}\right] \\
& \cdot \frac{t^{6 \alpha}}{\Gamma(1+2 \alpha)^{3}} \text {. }
\end{aligned}
$$

Solving the equation $D_{t}^{\alpha} \operatorname{Res}_{u, 2}(x, 0)=0$, we find that

$$
\begin{aligned}
f_{2}(x)= & f_{1 x}+6 f_{1} f_{x}^{2}+12 f f_{x} f_{1 x}+6 f f_{1} f_{x x} \\
& +3 f^{2} f_{1 x x}-f_{1 x x}-f_{1 x x x x}, \\
f_{2}(x)= & -\operatorname{sech}\left[\frac{x}{\sqrt{2}}\right]^{2} \tanh \left[\frac{x}{\sqrt{2}}\right] .
\end{aligned}
$$

To determine $f_{3}(x)$, we substitute

$$
\begin{aligned}
u_{3}(x, t)= & f(x)+f_{1}(x) \frac{t^{\alpha}}{\Gamma(1+\alpha)} \\
& +f_{2}(x) \frac{t^{2 \alpha}}{\Gamma(1+2 \alpha)}+f_{3}(x) \frac{t^{3 \alpha}}{\Gamma(1+3 \alpha)},
\end{aligned}
$$

into (43) where $\mathrm{k}=3$,

$$
\begin{aligned}
& \operatorname{Res}_{u, 3}(x, t)=f_{1}-f_{x}-6 f f_{x}^{2}-3 f^{2} f_{x x}+f_{x x} \\
& +f_{x x x x}+\left[f_{2}-f_{1 x}-12 f f_{x} f_{1 x}-6 f_{1} f_{x}^{2}\right. \\
& \left.+6 f f_{1} f_{x x}-3 f^{2} f_{1 x x}+f_{1 x x}+f_{1 x x x x}\right] \frac{t^{\alpha}}{\Gamma(1+\alpha)} \\
& +\left[f_{3}-f_{2 x}-12 f f_{x} f_{2 x}-6 f_{2} f_{x}^{2}-6 f f_{2} f_{x x}\right. \\
& \left.+3 f_{2 x x} f^{2}+f_{2 x x}+f_{2 x x x x}\right] \frac{t^{2 \alpha}}{\Gamma(1+2 \alpha)}+\left[-6 f f_{1 x}^{2}\right. \\
& \left.+12 f_{1} f_{x} f_{1 x}-3 f_{1}^{2} f_{x x}-6 f f_{1} f_{1 x x}\right] \frac{t^{2 \alpha}}{\Gamma(1+\alpha)^{2}} \\
& +\left[-12 f f_{x} f_{3 x}-f_{3 x}-6 f_{3} f_{x}^{2}-6 f f_{3} f_{x x}\right. \\
& +\frac{t^{4 \alpha}}{\Gamma(1+2 \alpha)^{2}}+\left[-12 f f_{1 x} f_{3 x}-12 f_{1} f_{x} f_{3 x}\right. \\
& + \\
& \left.+3 f^{2} f_{3 x x}+f_{3 x x}+f_{3 x x x x}\right] \frac{t^{3 \alpha}}{\Gamma(1+3 \alpha)} \\
& +\left[-12 f f_{1 x} f_{2 x}-12 f_{1} f_{x} f_{2 x}-12 f_{2} f_{x} f_{1 x}\right. \\
& +
\end{aligned}
$$




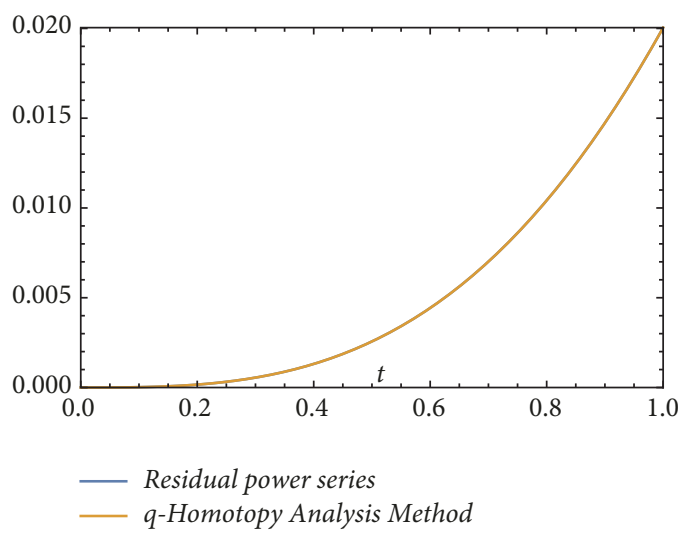

(a)

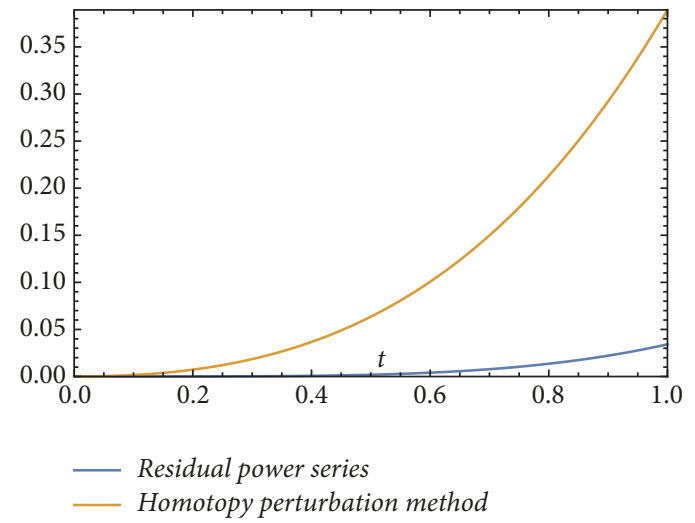

(b)

Figure 1: (a) Absolute errors of fractional Gardner equation, at $x=2, \varepsilon=1$. (b) Absolute errors of fractional Chan-Hilliard equation at $x=2$.

$$
\begin{aligned}
& \left.-3 f_{2}^{2} f_{1 x x}-6 f_{2 x x} f_{1} f_{2}\right] \frac{t^{5 \alpha}}{\Gamma(1+\alpha) \Gamma(1+2 \alpha)^{2}} \\
& +\left[-12 f f_{2 x} f_{3 x}-12 f_{2} f_{x} f_{3 x}-12 f_{3} f_{x} f_{2 x}\right. \\
& \left.-6 f_{2} f_{3} f_{x x}-6 f f_{3} f_{2 x x}-6 f f_{2} f_{3 x x}\right] \\
& \cdot \frac{t^{5 \alpha}}{\Gamma(1+2 \alpha) \Gamma(1+3 \alpha)}+\left[-12 f_{1} f_{1 x} f_{3 x}-6 f_{3} f_{1 x}^{2}\right. \\
& \left.-3 f_{1}^{2} f_{3 x x}-6 f_{1} f_{3} f_{1 x x}\right] \frac{t^{5 \alpha}}{\Gamma(1+\alpha)^{2} \Gamma(1+3 \alpha)} \\
& +\left[-12 f_{1} f_{2 x} f_{3 x}-12 f_{2} f_{1 x} f_{3 x}-12 f_{3} f_{1 x} f_{2 x}\right. \\
& \left.-6 f_{2} f_{3} f_{1 x x}-6 f_{1} f_{3} f_{2 x x}-6 f_{1} f_{2} f_{3 x x}\right] \\
& \cdot \frac{t^{6 \alpha}}{\Gamma(1+\alpha) \Gamma(1+2 \alpha) \Gamma(1+3 \alpha)}+\left[-6 f_{2} f_{2 x}^{2}\right. \\
& \left.-3 f_{2 x x} f_{2}^{2}\right] \frac{t^{6 \alpha}}{\Gamma(1+2 \alpha)^{3}}+\left[-6 f f_{3 x}^{2}-12 f_{3} f_{x} f_{3 x}\right. \\
& \left.-3 f_{3}^{2} f_{x x}-6 f f_{3} f_{3 x x}\right] \frac{t^{6 \alpha}}{\Gamma(1+3 \alpha)^{2}}+\left[-6 f_{1} f_{3 x}^{2}\right. \\
& \left.-12 f_{3} f_{1 x} f_{3 x}-3 f_{3}^{2} f_{1 x x}-6 f_{1} f_{3} f_{3 x x}\right] \\
& \cdot \frac{t^{7 \alpha}}{\Gamma(1+\alpha) \Gamma(1+3 \alpha)^{2}}+\left[-12 f_{2} f_{2 x} f_{3 x}-6 f_{3} f_{2 x}^{2}\right. \\
& \left.-6 f_{2} f_{3} f_{2 x x}-3 f_{2}^{2} f_{3 x x}\right] \frac{t^{7 \alpha}}{\Gamma(1+3 \alpha) \Gamma(1+2 \alpha)^{2}} \\
& +\left[-6 f_{2} f_{3 x}^{2}-12 f_{3} f_{2 x} f_{3 x}-3 f_{3}^{2} f_{2 x x}-6 f_{3 x x} f_{3} f_{2}\right] \\
& \cdot \frac{t^{8 \alpha}}{\Gamma(1+2 \alpha) \Gamma(1+3 \alpha)^{2}}+\left[-6 f_{3} f_{3 x}^{2}-3 f_{3}^{2} f_{3 x x}\right] \\
& \frac{t^{9 \alpha}}{\Gamma(1+3 \alpha)^{3}} \text {. }
\end{aligned}
$$

Applying $D_{t}^{2 \alpha}$ on both sides and then solving the equation $D_{t}^{2 \alpha} \operatorname{Res}_{u, 3}(x, 0)=0$, we get

$$
\begin{aligned}
& f_{3}(x)=\left[f_{2 x}+12 f f_{x} f_{2 x}+6 f_{2} f_{x}^{2}+6 f f_{2} f_{x x}\right. \\
& \left.+3 f_{2 x x} f^{2}-f_{2 x x}-f_{2 x x x x}\right]+\left[6 f f_{1 x}^{2}+12 f_{1} f_{x} f_{1 x}\right. \\
& \left.+3 f_{1}^{2} f_{x x}+6 f f_{1} f_{1 x x}\right] \frac{\Gamma(1+2 \alpha)}{\Gamma(1+\alpha)^{2}} \\
& f_{3}(x)=\frac{1}{8} \operatorname{sech}\left[\frac{x}{\sqrt{2}}\right]^{6}(-4 \sqrt{2} \\
& +(264-96 \cosh [\sqrt{2} x]+\sqrt{2} \sinh [2 \sqrt{2} x]) \\
& \left.\quad+\tanh \left[\frac{x}{\sqrt{2}}\right]\right)+\left(\frac{-21}{2} \operatorname{sech}\left[\frac{x}{\sqrt{2}}\right]^{6} \tanh \left[\frac{x}{\sqrt{2}}\right]\right. \\
& \left.+12 \operatorname{sech}\left[\frac{x}{\sqrt{2}}\right]^{4} \tanh \left[\frac{x}{\sqrt{2}}\right]^{3}\right) \frac{\Gamma(1+2 \alpha)}{\Gamma(1+\alpha)^{2}} .
\end{aligned}
$$

The solution in series form is given by

$$
\begin{aligned}
u(x, t)= & f(x)+f_{1}(x) \frac{t^{\alpha}}{\Gamma(1+\alpha)}+f_{2}(x) \frac{t^{2 \alpha}}{\Gamma(1+2 \alpha)} \\
& +f_{3}(x) \frac{t^{3 \alpha}}{\Gamma(1+3 \alpha)}+\ldots
\end{aligned}
$$

\section{Numerical Results}

This section deals with the approximate analytical solutions obtained by RPSM for Gardner and Cahn-Hilliard equations. In classical $\operatorname{case}(\alpha \longrightarrow 1)$, Figure 1 and Tables 1 and 2 describe the comparison between RPSM with qHAM [28] and HPM [36]. In fractional case, Figures 2, 3, and 4 describe the geometrical behavior of the solutions obtained by RPSM for different fractional value $\alpha$ of the two equations. 


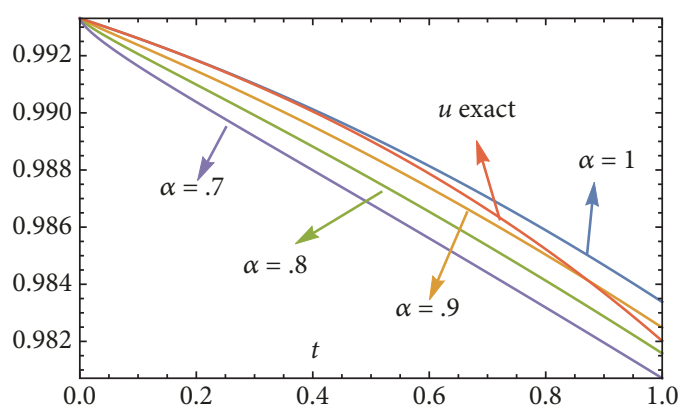

(a)

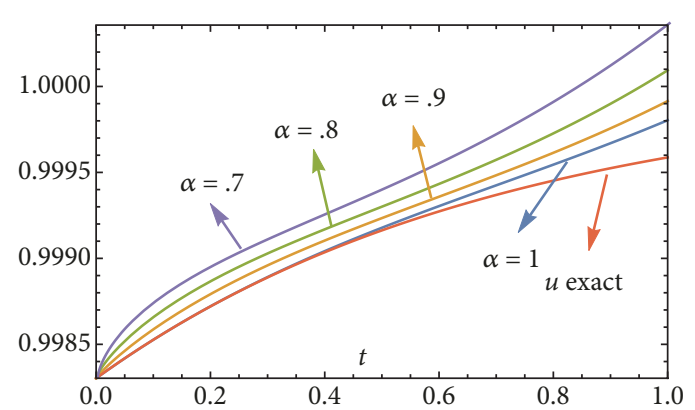

(b)

Figure 2: (a) Fractional Gardner equation at $x=5, \varepsilon=1$. (b) Fractional Chan-Hilliard equation at $x=5$.

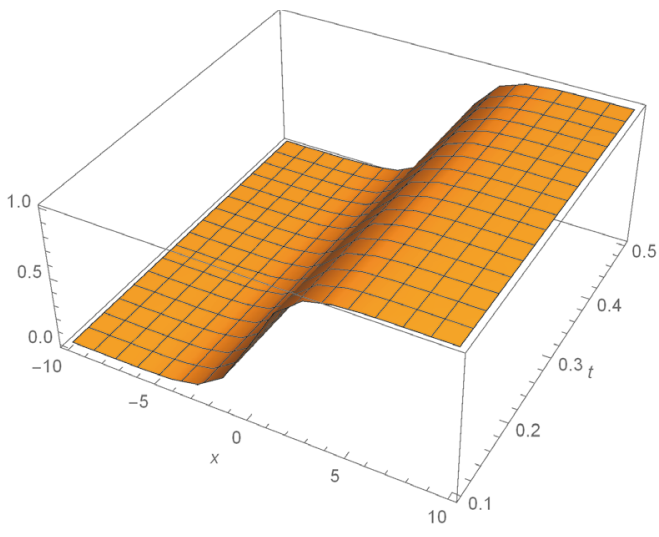

(a)

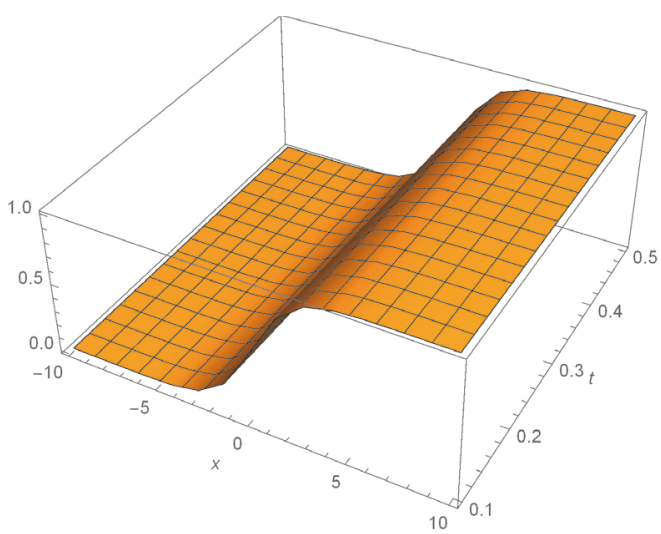

(c)

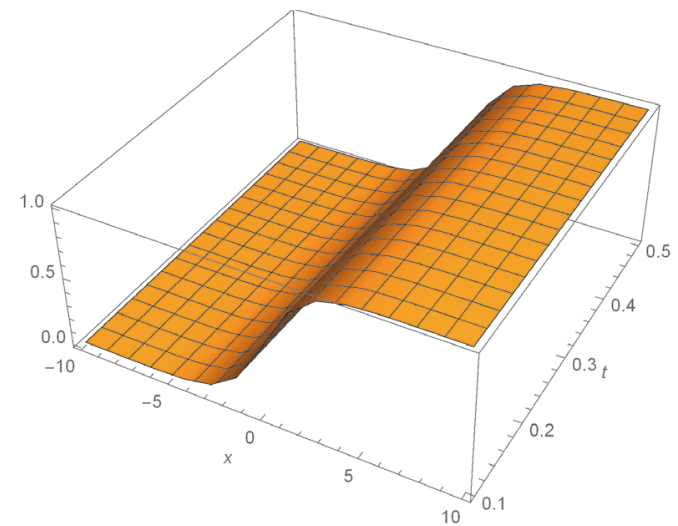

(b)

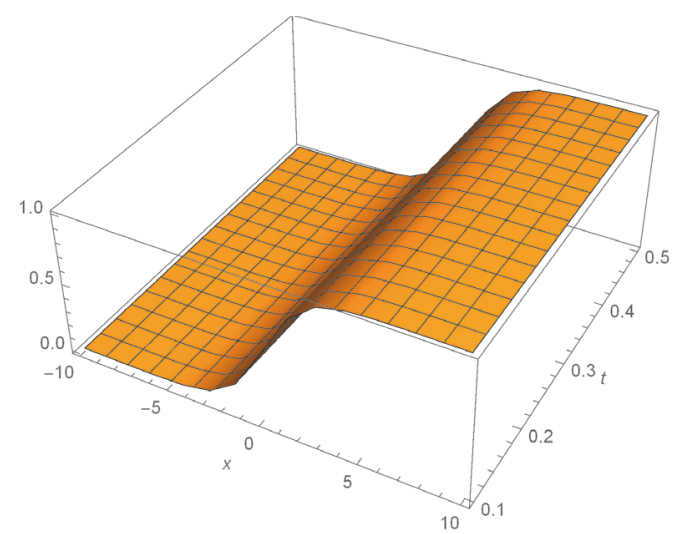

(d)

FIgURE 3: The approximate solution for fractional Gardner equation at $\varepsilon=1$ : (a) $\alpha=1$, (b) Exact solution, (c) $\alpha=.99$, and (d) $\alpha=.95$.

\section{Conclusions}

This work has used the RPSM for finding the solution of the time-fractional Gardner and Cahn-Hilliard equations. A very good agreement between the results obtained by the RPSM and q-HAM [28] was observed in Figure 1(a) and Table 1. Figure 1(b) and Table 2 indicate that the mentioned method achieves a higher level of accuracy than HPM [36]. Consequently, the work emphasized that the method introduces a significant improvement in this field over existing techniques.

\section{Data Availability}

[1] The [approximate solution obtained by q-homotopy analysis method] data used to support the findings of this study have been deposited in the [article] repository ([doi.org/10.1016/j.asej.2014.03.014]) [28]. [2] The [approximate solution obtained by homotopy perturbation method] data used to support the findings of this study have been deposited in the [article] repository ([doi.org/10.1080/ 10288457.2013.867627]) [36]. 


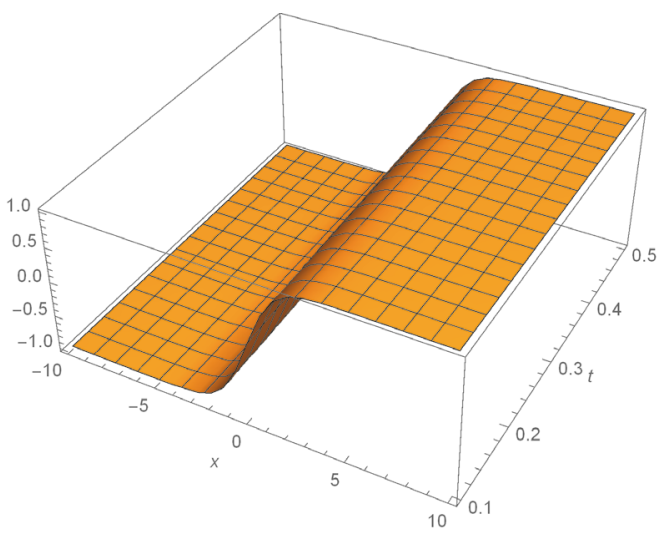

(a)

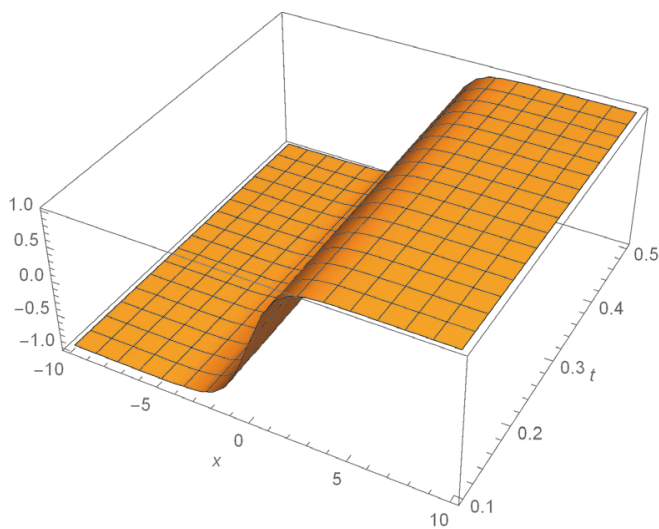

(c)

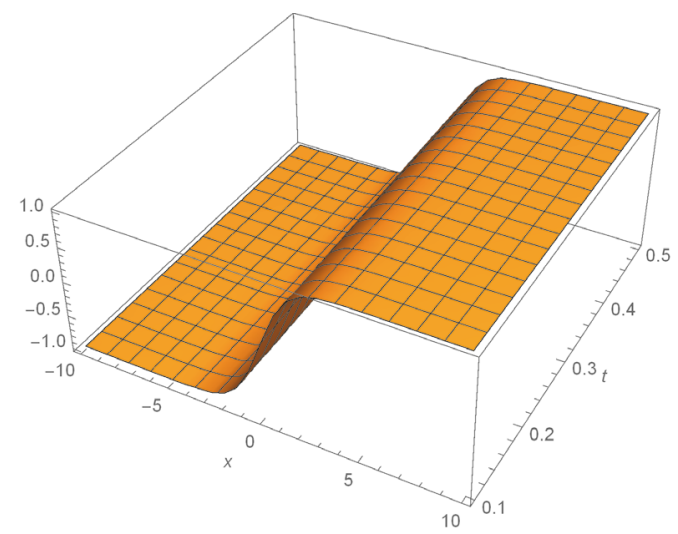

(b)

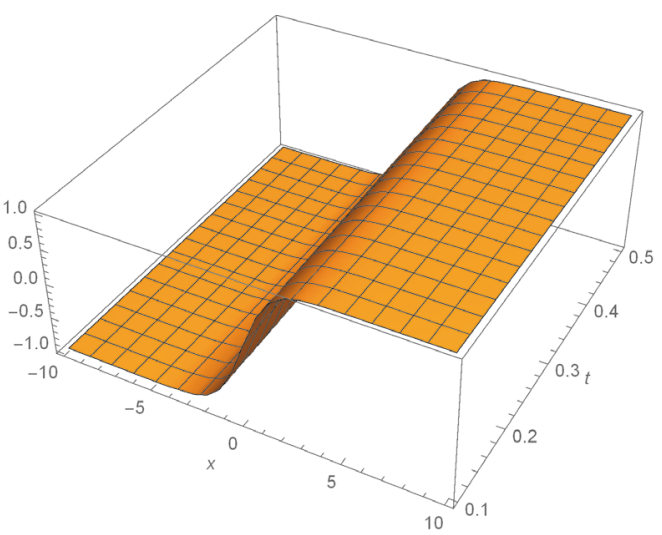

(d)

Figure 4: The approximate solution for fractional Chan-Hilliard equation: (a) $\alpha=1$, (b) Exact solution, (c) $\alpha=.99$, and (d) $\alpha=.95$.

TABLE 1: The absolute errors $\left|u_{\text {exact }}-u_{3}\right|$ for Gardner equation when $t=.2, \varepsilon=1, \alpha \longrightarrow 1$.

\begin{tabular}{ccc}
\hline $\mathrm{x}$ & $\left|u_{\text {exact }}-u_{R P S}\right|$ & $\left|u_{\text {exact }}-u_{q H A M}\right|$ \\
\hline .1 & $166.002 \times 10^{-6}$ & $166.002 \times 10^{-6}$ \\
.2 & $162.707 \times 10^{-6}$ & $162.707 \times 10^{-6}$ \\
.3 & $156.257 \times 10^{-6}$ & $156.257 \times 10^{-6}$ \\
.4 & $146.917 \times 10^{-6}$ & $146.917 \times 10^{-6}$ \\
.5 & $135.064 \times 10^{-6}$ & $135.064 \times 10^{-6}$ \\
\hline
\end{tabular}

TABLE 2: The absolute errors $\left|u_{\text {exact }}-u_{4}\right|$ for Cahn-Hilliard when $t=.2, \alpha \longrightarrow 1$.

\begin{tabular}{ccc}
\hline $\mathrm{x}$ & $\left|u_{\text {exact }}-u_{R P S}\right|$ & $\left|u_{\text {exact }}-u_{H P M}\right|$ \\
\hline .1 & $25.5541 \times 10^{-6}$ & $4.68338 \times 10^{-3}$ \\
.2 & $41.5291 \times 10^{-6}$ & $7.28902 \times 10^{-3}$ \\
.3 & $54.2246 \times 10^{-6}$ & $9.6162 \times 10^{-3}$ \\
.4 & $62.8898 \times 10^{-6}$ & $11.5931 \times 10^{-3}$ \\
.5 & $67.2637 \times 10^{-6}$ & $13.174 \times 10^{-3}$ \\
\hline
\end{tabular}

\section{Conflicts of Interest}

The authors declare that they have no conflicts of interest.

\section{References}

[1] L. Debnath, "Recent applications of fractional calculus to science and engineering," International Journal of Mathematics and Mathematical Sciences, no. 54, pp. 3413-3442, 2003.

[2] M. Rahimy, "Applications of fractional differential equations," Applied Mathematical Sciences, vol. 4, no. 49-52, pp. 2453-2461, 2010.

[3] A. A. Arafa and S. Z. Rida, "Numerical solutions for some generalized coupled nonlinear evolution equations," Mathematical and Computer Modelling, vol. 56, no. 11-12, pp. 268-277, 2012.

[4] A. A. Arafa, "Series solutions of time-fractional host-parasitoid systems," Journal of Statistical Physics, vol. 145, no. 5, pp. 13571367, 2011.

[5] N. H. Sweilam, M. M. Khader, and R. F. Al-Bar, "Numerical studies for a multi-order fractional differential equation," Physics Letters A, vol. 371, no. 1-2, pp. 26-33, 2007.

[6] A. Golbabai and K. Sayevand, "Fractional calculus- a new approach to the analysis of generalized fourth-order diffusionwave equations," Computers \& Mathematics with Applications. An International Journal, vol. 61, no. 8, pp. 2227-2231, 2011.

[7] K. A. Gepreel, “The homotopy perturbation method applied to the nonlinear fractional Kolmogorov-Petrovskii-PISkunov equations," Applied Mathematics Letters, vol. 24, no. 8, pp. 14281434, 2011.

[8] A. A. Arafa, S. Z. Rida, and H. Mohamed, "Approximate analytical solutions of Schnakenberg systems by homotopy 
analysis method," Applied Mathematical Modelling: Simulation and Computation for Engineering and Environmental Systems, vol. 36, no. 10, pp. 4789-4796, 2012.

[9] A. A. Arafa, S. Z. Rida, and M. Khalil, "The effect of anti-viral drug treatment of human immunodeficiency virus type 1 (HIV1) described by a fractional order model," Applied Mathematical Modelling: Simulation and Computation for Engineering and Environmental Systems, vol. 37, no. 4, pp. 2189-2196, 2013.

[10] M. Javidi, "A numerical solution of the generalized BurgersHuxley equation by spectral collocation method," Applied Mathematics and Computation, vol. 178, no. 2, pp. 338-344, 2006.

[11] A.-M. Wazwaz, "Analytic study on Burgers, Fisher, Huxley equations and combined forms of these equations," Applied Mathematics and Computation, vol. 195, no. 2, pp. 754-761, 2008.

[12] X.-W. Zhou, "Exp-function method for solving Huxley equation," Mathematical Problems in Engineering, Art. ID 538489, 7 pages, 2008.

[13] A. A. Arafa, S. Z. Rida, A. A. Mohammadein, and H. M. Ali, "Solving nonlinear fractional differential equation by generalized Mittag-Leffler function method," Communications in Theoretical Physics, vol. 59, no. 6, pp. 661-663, 2013.

[14] M. Sari and G. Gürarslan, "Numerical solutions of the generalized Burgers-Huxley equation by a differential quadrature method," Mathematical Problems in Engineering, Art. ID 370765, 11 pages, 2009.

[15] O. Abu Arqub, "An iterative method for solving fourth-order boundary value problems of mixed type integro-differential equations," Journal of Computational Analysis and Applications, vol. 18, no. 5, pp. 857-874, 2015.

[16] O. Abu Arqub, "Fitted reproducing kernel Hilbert space method for the solutions of some certain classes of time-fractional partial differential equations subject to initial and Neumann boundary conditions," Computers \& Mathematics with Applications, vol. 73, no. 6, pp. 1243-1261, 2017.

[17] A.-M. Wazwaz, "Solitons and singular solitons for the GardnerKP equation," Applied Mathematics and Computation, vol. 204, no. 1, pp. 162-169, 2008.

[18] C. S. Gardner, J. M. Greene, M. D. Kruskal, and R. M. Miura, "Method for solving the Korteweg-deVries equation," Physical Review Letters, vol. 19, no. 19, pp. 1095-1097, 1967.

[19] A.-M. Wazwaz, "New solitons and kink solutions for the Gardner equation," Communications in Nonlinear Science and Numerical Simulation, vol. 12, no. 8, pp. 1395-1404, 2007.

[20] Z. Fu, S. Liu, and S. Liu, "New kinds of solutions to Gardner equation," Chaos, Solitons \& Fractals, vol. 20, no. 2, pp. 301-309, 2004.

[21] G.-q. Xu, Z.-b. Li, and Y.-p. Liu, "Exact solutions to a large class of nonlinear evolution equations," Chinese Journal of Physics, vol. 41, no. 3, pp. 232-241, 2003.

[22] D. Baldwin, Ü. Göktas, W. Hereman, L. Hong, R. S. Martino, and J. C. Miller, "Symbolic computation of exact solutions expressible in hyperbolic and elliptic functions for nonlinear PDEs," Journal of Symbolic Computation, vol. 37, no. 6, pp. 669705, 2004.

[23] W. Hereman and A. Nuseir, "Symbolic methods to construct exact solutions of nonlinear partial differential equations," Mathematics and Computers in Simulation, vol. 43, no. 1, pp. 1327, 1997.
[24] Y. Ugurlu and D. g. Kaya, "Solutions of the Cahn-Hilliard equation," Computers \& Mathematics with Applications. An International Journal, vol. 56, no. 12, pp. 3038-3045, 2008.

[25] J. W. Cahn and J. E. Hilliard, "Free energy of a nonuniform system. I. Interfacial free energy," The Journal of Chemical Physics, vol. 28, no. 2, pp. 258-267, 1958.

[26] S. M. Choo, S. K. Chung, and Y. J. Lee, "A conservative difference scheme for the viscous Cahn-Hilliard equation with a nonconstant gradient energy coefficient," Applied Numerical Mathematics, vol. 51, no. 2-3, pp. 207-219, 2004.

[27] M. E. Gurtin, "Generalized Ginzburg-Landau and CahnHilliard equations based on a microforce balance," Physica D: Nonlinear Phenomena, vol. 92, no. 3-4, pp. 178-192, 1996.

[28] O. S. Iyiola and O. G. Olayinka, "Analytical solutions of timefractional models for homogeneous Gardner equation and nonhomogeneous differential equations," Ain Shams Engineering Journal, vol. 5, no. 3, pp. 999-1004, 2014.

[29] Y. Pandir and H. H. Duzgun, "New Exact Solutions of Time Fractional Gardner Equation by Using New Version of FExpansion Method," Communications in Theoretical Physics, vol. 67, no. 1, pp. 9-14, 2017.

[30] J. Ahmad and S. T. Mohyud-Din, "An efficient algorithm for some highly nonlinear fractional PDEs in mathematical physics," PLoS ONE, vol. 9, no. 12, Article ID e109127, 2014.

[31] W. Li, H. Yang, and B. He, "Exact solutions of fractional Burgers and Cahn-Hilliard equations using extended fractional Riccati expansion method," Mathematical Problems in Engineering, Art. ID 104069, 9 pages, 2014.

[32] H. Jafari, H. Tajadodi, N. Kadkhoda, and D. Baleanu, "Fractional subequation method for Cahn-Hilliard and KleinGordon equations," Abstract and Applied Analysis, vol. 2013, Article ID 587179, 5 pages, 2013.

[33] M. S. Mohamed and K. S. Mekheimer, "Analytical approximate solution for nonlinear space-time fractional Cahn-Hilliard equation," International Electronic Journal of Pure and Applied Mathematics, vol. 7, no. 4, pp. 145-159, 2014.

[34] Z. Dahmani and M. Benbachir, "Solutions of the Cahn-Hilliard equation with time- and space-fractional derivatives," International Journal of Nonlinear Science, vol. 8, no. 1, pp. 19-26, 2009.

[35] D. Baleanu, Y. Ugurlu, M. Inc, and B. Kilic, "Improved ( G' / G ) -Expansion Method for the Time-Fractional Biological Population Model and Cahn-Hilliard Equation," Journal of Computational and Nonlinear Dynamics, vol. 10, no. 5, p. 051016, 2015.

[36] A. Bouhassoun and M. Hamdi Cherif, "Homotopy Perturbation Method For Solving The Fractional Cahn-Hilliard Equation," Journal of Interdisciplinary Mathematics, vol. 18, no. 5, pp. 513524, 2015.

[37] J. Ahmad and S. T. Mohyud-Din, "An efficient algorithm for nonlinear fractional partial differential equations," Proceedings of the Pakistan Academy of Sciences, vol. 52, no. 4, pp. 381-388, 2015.

[38] J. Manafian and M. Lakestani, "A new analytical approach to solve some of the fractional-order partial differential equations," Indian Journal of Physics, vol. 91, no. 3, pp. 243-258, 2017.

[39] S. Tuluce Demiray, Y. Pandir, and H. Bulut, "Generalized Kudryashov method for time-fractional differential equations," Abstract and Applied Analysis, Art. ID 901540, 13 pages, 2014.

[40] O. Abu Arqub, "Series solution of fuzzy differential equations under strongly generalized differentiability," Journal of Advanced Research in Applied Mathematics, vol. 5, no. 1, pp. 3152, 2013. 
[41] O. Abu Arqub, A. El-Ajou, A. S. Bataineh, and I. Hashim, "A representation of the exact solution of generalized Lane-Emden equations using a new analytical method," Abstract and Applied Analysis, Art. ID 378593, 10 pages, 2013.

[42] O. Abu Arqub, Z. Abo-Hammour, R. Al-Badarneh, and S. Momani, "A reliable analytical method for solving higher-order initial value problems," Discrete Dynamics in Nature and Society, vol. 2013, Article ID 673829, 12 pages, 2013.

[43] A. El-Ajou, O. Abu Arqub, and S. Momani, "Approximate analytical solution of the nonlinear fractional KdV-Burgers equation: a new iterative algorithm," Journal of Computational Physics, vol. 293, pp. 81-95, 2015.

[44] O. A. Arqub, A. El-Ajou, and S. Momani, "Constructing and predicting solitary pattern solutions for nonlinear timefractional dispersive partial differential equations," Journal of Computational Physics, vol. 293, pp. 385-399, 2015.

[45] O. A. Arqub, A. El-Ajou, Z. A. Zhour, and S. Momani, "Multiple solutions of nonlinear boundary value problems of fractional order: A new analytic iterative technique," Entropy, vol. 16, no. 1, pp. 471-493, 2014.

[46] A. El-Ajou, O. Abu Arqub, S. Momani, D. Baleanu, and A. Alsaedi, "A novel expansion iterative method for solving linear partial differential equations of fractional order," Applied Mathematics and Computation, vol. 257, pp. 119-133, 2015.

[47] A. El-Ajou, O. Abu Arqub, Z. Al Zhour, and S. Momani, "New results on fractional power series: theories and applications," Entropy. An International and Interdisciplinary Journal of Entropy and Information Studies, vol. 15, no. 12, pp. 5305-5323, 2013.

[48] A. El-Ajou, O. Abu Arqub, and M. Al-Smadi, "A general form of the generalized Taylor's formula with some applications," Applied Mathematics and Computation, vol. 256, pp. 851-859, 2015. 


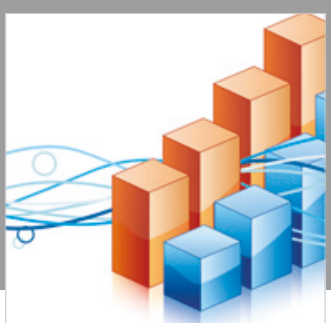

Advances in

Operations Research

\section{-n-m}
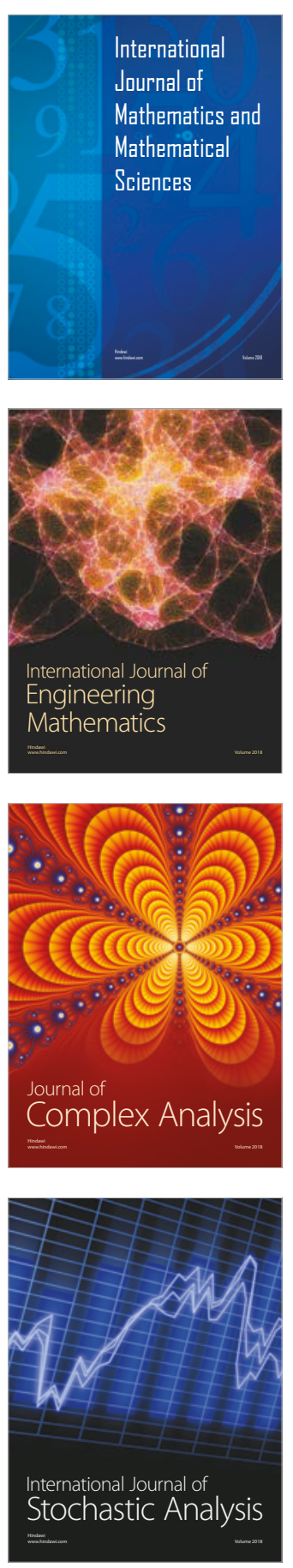
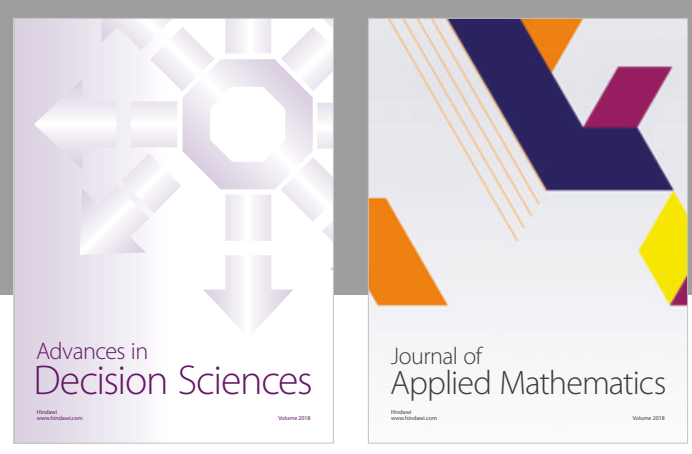

Journal of

Applied Mathematics
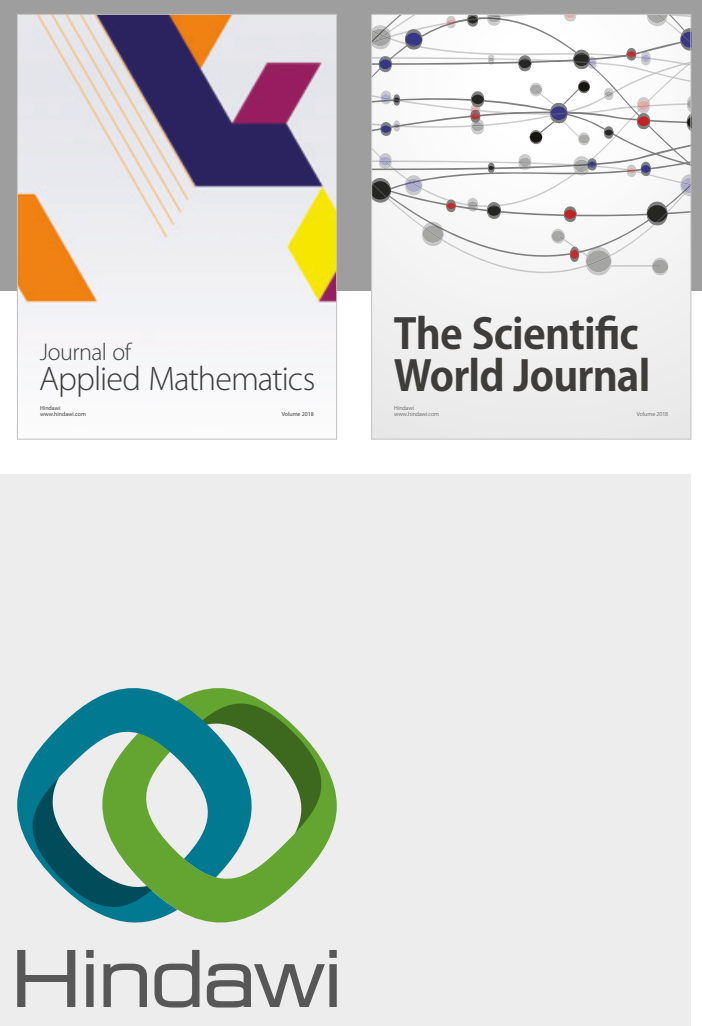

Submit your manuscripts at

www.hindawi.com

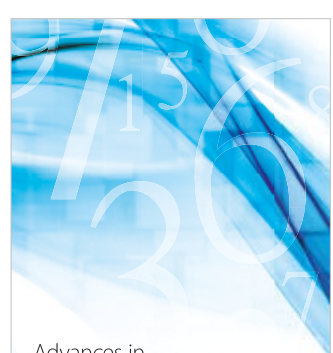

Advances in
Numerical Analysis
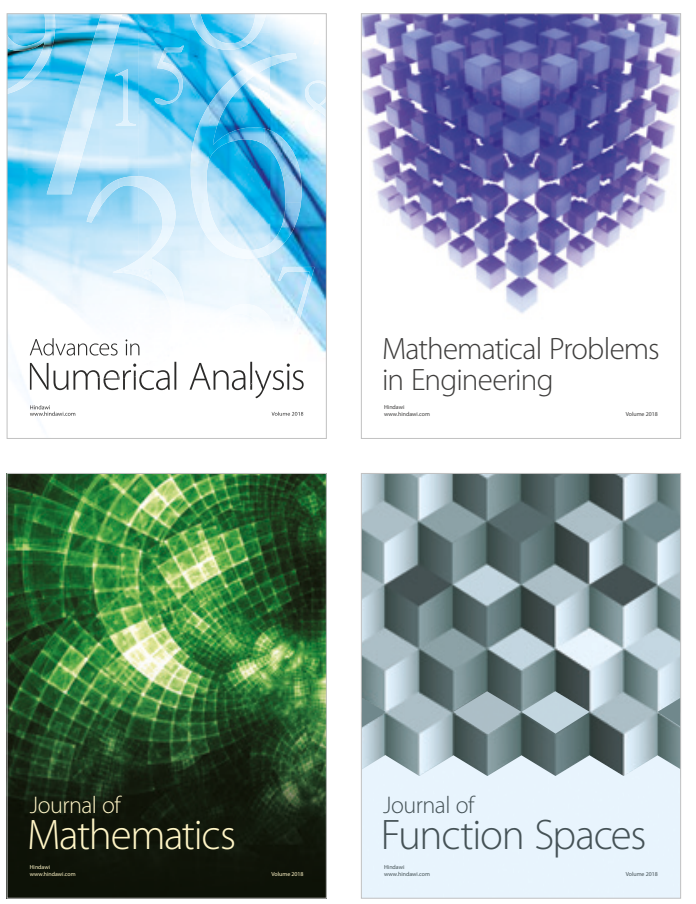

Mathematical Problems in Engineering

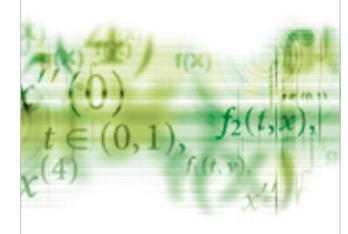

International Journal of

Differential Equations

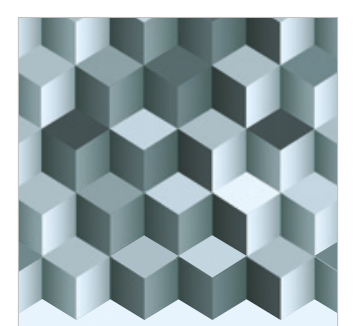

Journal of

Function Spaces

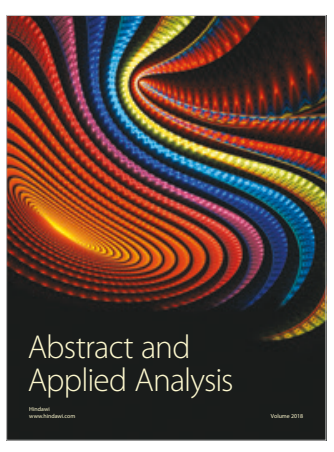

The Scientific

World Journal

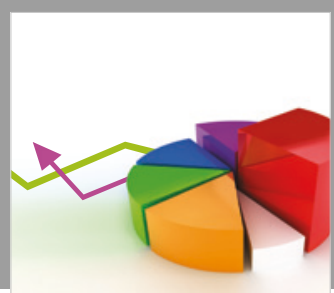

Journal of

Probability and Statistics
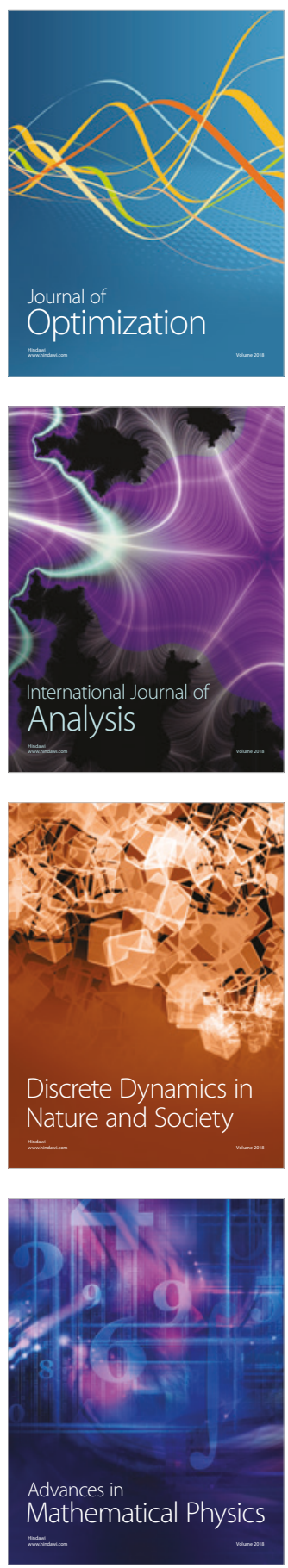